\title{
O enfermeiro frente às práticas integrativas e complementares em saúde na estratégia de saúde da família
}

The nurse against integrative and complementary practices in health in the family health strategy

El enfermero frente a las prácticas integrativas y complementarios en salud en la estrategia de salud de la familia

Juliane Rosalia de Almeida ${ }^{1 *}$, Márcia Carolina dos Santos Vianini ${ }^{1}$, Danila Maria Silva ${ }^{1}$, Rodolfo Almeida Meneghin ${ }^{1}$, Gilberto de Souza ${ }^{1}$, Márcio Antônio Resende¹.

\section{RESUMO}

Objetivo: Almejando uma melhoria na qualidade do processo de cuidados em Enfermagem que esta pesquisa tem por objetivo mostrar que as técnicas Integrativas e Complementares em saúde podem ser associadas aos cuidados do profissional enfermeiro. Métodos: Em suma, este trabalho consiste em um estudo exploratório descritivo de revisão Bibliográfica com uma abordagem qualitativa a qual permite refletir sobre a importância da inserção das práticas integrativas e complementares no processo de cuidados da enfermagem valorizando o modo de pensar e agir do enfermeiro fazendo jus o seu processo de aprendizagem. Resultados e Discussão: As práticas alternativas e complementares em saúde são técnicas que envolvem e visam estimular mecanismos naturais de prevenção de agravos e recuperação da saúde. Tais práticas devem ser bem exercidas pelo profissional enfermeiro principalmente em uma Unidade Básica de saúde, sendo esta considerada a porta de entrada do atendimento onde a oferta e procura de cuidados é alarmante. Estudos científicos demonstraram que ainda há profissionais enfermeiros atuantes em unidade básica de saúde com um déficit na atuação frente às práticas alternativas e complementares em saúde. Considerações finais: Nosso levantamento evidenciou a necessidade de incrementar as práticas interativas complementares às práticas de enfermagem, pois a realidade do atendimento em saúde, atualmente, ainda necessita de um cuidado individualizado, humanizado e qualificado ao usuário que necessita de cuidado e apoio.

Palavras-chaves: Práticas Integrativas e Complementares; Enfermeiro; Saúde.

\begin{abstract}
Objective: This fact aroused interest in developing this work in order to enable nurses to rethink their care process. Aiming for an improvement in the quality of the Nursing care process, this research aims to show that Integrative and Complementary health techniques can be associated to the care of the nursing professional. Methods: In short, this work consists of an exploratory descriptive study of Bibliographic review with a qualitative approach that allows to reflect on the importance of the insertion of integrative and complementary practices in the process of nursing care, valuing the way of thinking and acting of the nurse doing the right learning process. Results and Discussion: Alternative and complementary health practices are techniques that involve and aim to stimulate natural mechanisms of disease prevention and health recovery. Such practices should be well performed by the nurse practitioner, especially in a Basic Health Unit, which is considered the gateway to care where the supply and demand for care is alarming. Scientific studies have shown that there are still professional nurses working in the basic health unit with a deficit in the performance of alternative and complementary health practices. Conclusion: Our survey highlights the need to increase therapeutic practices in nursing, health care, health care, health care, health care, and health care.
\end{abstract}

Keywords: Integrative and Complementary Practices; Nurse; Cheers.

\footnotetext{
${ }^{1}$ Centro Universitário Presidente Tancredo de Almeida Neves (UNIPTAN).

*E-mail: jullyalmeida1@hotmail.com
} 


\section{RESUMEN}

Objetivo: Alineando una mejora en la calidad del proceso de cuidados en Enfermería que esta investigación tiene por objetivo mostrar que las técnicas Integrativas y Complementarias en salud pueden ser asociadas a los cuidados del profesional enfermero. Metodos: En resumen, este trabajo consiste en un estudio exploratorio descriptivo de revisión Bibliográfica con un abordaje cualitativo que permite reflexionar sobre la importancia de la inserción de las prácticas integrativas y complementarias en el proceso de cuidados de la enfermería valorizando el modo de pensar y actuar del enfermero haciendo justicia su proceso de aprendizaje. Resultados e Discusión: Las prácticas alternativas y complementarias en salud son técnicas que involucran y apuntan a estimular mecanismos naturales de prevención de agravios y recuperación de la salud. Tales prácticas deben ser bien ejercidas por el profesional enfermeiro principalmente en una Unidad Básica de salud, siendo ésta considerada la puerta de entrada de la atención donde la oferta y demanda de cuidados es alarmante. Estudios científicos han demostrado que todavía hay profesionales enfermeros que actúan em unidad básica de salud con un déficit en la actuación frente a las prácticas alternativas y complementarias en salud. Consideraciones finales: Nuestro levantamiento evidencia la necesidad de incrementar las prácticas terapéuticas de enfermería, atención sanitaria, atención sanitaria, atención sanitaria, atención sanitaria y asistencia sanitaria.

Palabras claves: Prácticas Integrativas y Complementarias; Enfermero; Salud.

\section{INTRODUÇÃO}

Este trabalho tem a discorrer sobre o enfermeiro frente às práticas integrativas e complementares em saúde, na estratégia de saúde da família. Assim, é necessário deixar explícito neste artigo o que são essas técnicas e como elas podem ser associados aos cuidados do profissional enfermeiro que assiste o paciente que busca por esses cuidados na Estratégia de Saúde da Família.

As práticas Integrativa e Complementares (PIC) devem ser bem exercidas pelo profissional enfermeiro principalmente em uma Unidade Básica de Saúde, sendo esta considerada a porta de entrada do atendimento onde a oferta e procura de cuidados é alarmante (CAMPOS, 2014).

A Organização Mundial em Saúde (OMS), por meio do programa de medicina tradicional vem incentivando há vários anos o uso de PIC em todo mundo. As abordagens da OMS culminaram na criação de um documento normativo visando fortalecer políticas para o uso racional, moderado e integrado nas terapias não ortodoxias no sistema nacional de atenção à saúde (BRASIL, 2017).

A atuação do enfermeiro na Estratégia Saúde da Família (ESF) se dá em três diferentes processos de trabalhos, a saber, assistência, gerência e educação. A complexidade de tal processo vem sendo reconhecida de maneira gradativa no Brasil (BRASIL, 2017). Pode-se destacar que o trabalho do enfermeiro da ESF apresenta outras particularidades com relação às demais profissões, além de ser responsável pela assistência, também participa na gestão do processo de trabalho de outros colaboradores em enfermagem, dos agentes comunitários de saúde, bem como pela educação permanente destes. (THIAGO, TESSER, 2010).

A Estratégia Saúde da Família, é um programa desenvolvido pelo governo, dentro das unidades básicas de saúde contam com uma equipe multidisciplinar, médico, enfermeiro, dentista, agentes de saúde, técnico de enfermagem, e podem ou não contar com médicos e enfermeiros especialistas em saúde pública (BRASIL, 2017).

O fortalecimento da política nacional de práticas integrativas e complementares em saúde tem proporcionado o aumento à procura de tais serviços, por parte dos usuários do Sistema Único de Saúde (SUS) (EMÍLIO, et al., 2014).

No que concerne à importância do papel do profissional enfermeiro na Estratégia saúde da Família, que o objetivo deste trabalho é mostrar que as técnicas Integrativas e Complementares em saúde podem ser associadas aos cuidados do profissional enfermeiro. 
O papel do profissional enfermeiro na Estratégia saúde da Família, onde o é proposto por este trabalho uma reflexão ao leitor de quão é importante à capacitação desse profissional que está na linha de frente do cuidado para que as práticas integrativas e complementares sejam inseridas e desenvolvidas no processo do cuidado.

\section{METODOLOGIA}

Trata-se de um estudo exploratório, descritivo, de revisão bibliográfica com abordagem qualitativa que atenderá o objetivo proposto neste artigo. O levantamento bibliográfico foi por meio de pesquisas embasados no Ministério da Saúde (MS) e Biblioteca Virtual em Saúde (BVS) e foi realizado busca nas seguintes bases de dados de Literatura, Latino-americana em Ciências da Saúde (LILACS), Scientific Electronic Library Online (SciELO.) Como critérios de inclusão foi utilizados relatos de experiências relativo ao tema nos últimos 20 anos tendo como objetivo incentivar as práticas Integrativas e Complementares em Saúde na ESF.

\section{RESULTADOS E DISCUSSÃO}

\section{Práticas Integrativas e Complementares em Saúde}

As PIC em Saúde envolvem técnicas de abordagens com o objetivo de incitar mecanismos naturais de prevenção a agravos e recuperação da saúde através de tecnologias eficazes, seguras e leves, por meio da criação de vínculo terapêutico, na integração do indivíduo ao autocuidado (THIAGO, TESSER, 2010).

As terapias complementares fazem parte de uma abordagem ao paciente de forma integral, uma abordagem holística e natural da saúde podendo ser relatada como práticas que pertencem ao patrimônio cultural e ao inconsciente coletivo da humanidade, as práticas não convencionais em saúde se utilizam de recursos terapêuticos com eficácia comprovada, que complementam as terapias convencionais, sempre levados em conta à individualidade de cada pessoa, abordando técnicas seguras, com responsabilidade pautada pelo profissional (BARBOSA, et. al, 2011).

O homem possui mais do que um corpo físico, possui mente e espírito, que não deve ser dissociado, e necessita de cuidado como um todo, o homem holístico é formado por vários fatores e espectro de sistemas de energias interativas a vários fatores como emoção, nutrição, estressantes de natureza física e sono insuficiente, tornando o homem susceptível à doença (FONTANELLA, et. al, 2016).

A OMS, por meio do programa de medicina tradicional vem incentivando há vários anos o uso de práticas integrativas e complementares em todo mundo. As abordagens da OMS culminaram na criação de um documento normativo visando fortalecer políticas para o uso racional, moderado e integrado nas terapias não ortodoxias no sistema nacional de atenção a saúde (BRASIL, 2017).

A visão biomédica enxerga o corpo humano de forma fragmentado e presta assistência de forma dissociada de maneira especifica. Desta forma induzem os profissionais em saúde negligenciarem aspectos psicossociais, emocionais, espirituais, culturais e ambientais do indivíduo, afastando-se do cuidado integral e holístico tão defendido atualmente (FONTANELLA, et. al, 2016).

O modelo biomédico em saúde mostra constantemente possuir limitações em interagir com outras dimensões do ser humano, por fragmentar o cuidado, interferindo na qualidade de vida do paciente, cada vez mais tem se observado um modelo de atenção que abrange de forma integral o paciente que visa à fundamentação de uma assistência de qualidade que reconhece as articulações e integralidades do paciente (PENNAFORT, et. al, 2012).

É válido destacar que a relação entre o cuidado ético e de qualidade, é independente do modelo de cuidar ofertado pelo profissional. No entanto, as características próprias da biomedicina e de suas formas de cuidar apelam para objetividade, dificultam o processo de participação da equipe multidisciplinar, em contra ponto o profissional de saúde quando opta em praticar PIC no cuidado, deve ter a familiarização com uma de suas bases fundantes (ISLÂNDIA, et. al, 2012). 
Com o intuito de garantir à integralidade na atenção a saúde no SUS ,foi estabelecida em forma de portaria Ministerial o 971 em 03 de maio de 2006, e oㅜ 1.600, de 17 de julho de 2006, a Política Nacional de Práticas Integrativas e Complementares a qual regulamenta essas práticas no Sistema Único de Saúde (BRASIL, 2006).

Segundo o Parecer Normativo no 004/95 algumas terapias alternativas são reconhecidas pelo COFEN, como por exemplo, a Acupuntura, Iridologia, Fitoterapia, Reflexologia, Quiropraxia e Massoterapia, tais terapias têm suas origens em culturas orientais, não sendo, portanto, sua aplicação privativa a nenhuma categoria profissional específica (BRASIL, 2010).

No Brasil as discussões em volta da Política Nacional Integrativa Complementar (PNIC), tiveram inicio na década de 1980 a VIII junto a Conferencia Nacional de Saúde (CNS), em 1986 que trouxe um conceito amplo de saúde visando, promoção, prevenção, proteção e recuperação sendo estas as referencias na construção do SUS, nesta conferencia foi integrada ao relatório, a introdução PIC no âmbito dos serviços de saúde, ofertando ao usuário o acesso democrático para escolher a terapêutica preferida junto à equipe multidisciplinar (Brasil, 2006).

\section{O SUS é as práticas Integrativas em Saúde}

A institucionalização da Política Nacional Integrativa Complementar no Sistema Único de Saúde é um desafio para os gestores públicos que contam com recursos reduzidos, como recursos humanos e financeiros uma vez que a falta de diretrizes para esta implantação dificulta a consolidação das práticas, especialmente na atenção primária (MELO 2013).

O fortalecimento da política nacional de práticas integrativas e complementares em saúde tem proporcionado o aumento por procura de tais serviços, por parte dos usuários do SUS. No que concerne à prevenção de agravos e promoção à saúde relacionada ao usuário do SUS que necessita de cuidado humanizado, a PNPIC vem contribuir para sustentar e valorizar os princípios do SUS (THIAGO, TESSER, 2010).

A integralidade do cuidado à saúde é prescrita como diretriz pela $8^{\underline{a}}$ Conferência Nacional de Saúde e padronizada como princípio e doutrina do Sistema Único de Saúde, direciona para a oferta dessas práticas no Brasil. Quais podem ajudar a desmedicalização parcial do cuidado profissional, além de serem socialmente valorizadas e desejadas (THIAGO, TESSER, 2010).

A Conferência Nacional de Saúde da década de 80 indicou demanda quanto à institucionalização das práticas integrativas e complementares em saúde, e emitiu em seu relatório final que se implantassem as práticas alternativas e complementares em saúde como forma de assegurar a autonomia ao cliente no momento da escolha de sua opção terapêutica fazendo valer os princípios doutrinários do SUS (Brasil, 2009).

A primeira diretriz conceitua a estruturação e fortalecimento da atenção em PIC no SUS, perante sua inserção, em todos os níveis de atenção dando preferência na atenção básica.

Assim, a segunda diretriz se refere à expansão de meios de qualificação em PIC para profissionais do SUS, de acordo com os princípios e diretrizes estabelecidos para a educação permanente. A terceira diretriz informa as ações de divulgação e informação dos conhecimentos das PICS para os profissionais de saúde e usuários gestores do SUS (Brasil, 2006).

A atenção primária desenvolve o papel de indicativo de saúde, de uma população, pois desempenha a função de porta de entrada ao sistema único de saúde, ofertando seus serviços próximos às moradias dos seus clientes. A atenção primária consegue solucionar $80 \%$ dos problemas de saúde de sua área de abrangência, assim exercendo seus princípios de prevenção, promoção e recuperação à saúde (LIMA, 2012).

A Estratégia e Saúde da Família, programa desenvolvido pelo governo, dentro das unidades básicas de saúde conta com uma equipe multidisciplinar, médico, enfermeiro, dentista, agentes de saúde, técnico de enfermagem, e podem ou não contar com médicos e enfermeiros especialistas (AZEVEDO, PELICIONI, 2011). 
A área de atuação das PICs em saúde aborda sistemas médicos complexos e recursos terapêuticos, os quais denominados pela ORGANIZAÇÃO MUNDIAL DE SAÚDE de medicina tradicional e complementar /alternativa (MT/MCA) estas abordagens visam a estimular os meios naturais de prevenção de agravos e recuperação em saúde por meios de tecnologias eficazes, seguras e leves (BRASIL, 2017).

Todas as intervenções decorrentes das políticas nacionais voltadas à integração das PICs ao SUS, especialmente quando se utilizam plantas medicinais e derivadas como meios terapêuticos perpassam pelo entendimento e valorização da multiculturalidade e interculturalidade, por gestores e profissionais de saúde, para melhor equidade e integralidade da atenção (BRASIL, 2017).

Estima-se que PICS retratam o contraponto ao modelo biologizante e medicalizante à medida que enfatizam o cuidado à promoção da saúde. Torna-se necessário desencadear processos educativos permanentes que assegurem principalmente o profissional enfermeiro à formação e informação contínua visando profissionais de saúde capacitados em PICS (EMÍLIO, et. al, 2014).

\section{0 enfermeiro frente às práticas integrativas e complementares em saúde}

Dentre os profissionais de saúde, os enfermeiros são os que mantêm contato maior com os usuários dos serviços de saúde, tendo grande potencial para detectar os problemas relacionados e desenvolver ações assistenciais (ISLÂNDIA, et. al, 2012).

Segundo Melo (2013), a atuação do enfermeiro na Estratégia saúde da Família, se dá pelo menos três processos de trabalho diferentes: assistência, gerenciamento e educação. Ambos os processos são desenvolvidos de maneira cíclica visando uma boa opinião com relação aos cuidados oferecidos aos pacientes.

De acordo com Campos (2014) a atuação do enfermeiro na Unidade de Atenção Básica, em especial, na estratégia Saúde da Família não está limitada apenas nesses três processos, indo além do que a comunidade possa vislumbrar. Ele se responsabiliza pela gestão do processo de trabalho dos agentes comunitários de saúde, pela educação permanente de todos os profissionais atuantes na Estratégia Saúde da Família, trabalhando em equipe e nas ações de educação em saúde.

Reforçando os dizeres do autor supracitado que Junior et.al. (2016) demonstra em seu artigo que o papel do profissional enfermeiro refletem em uma pluralidade de atividades que justifica a interação das práticas integrativas e complementares na assistência deste profissional. Uma vez inseridas essas práticas na assistência do enfermeiro ele conseguirá visualizar o paciente como um todo, cuidar e assistir integralmente o ser humano de forma holística. Passará avaliar não somente a doença, mas o indivíduo doente e facilitará na detecção do diagnóstico de enfermagem como no planejamento das intervenções que serão aplicados aos pacientes assistidos.

Considerando a aproximação da enfermagem e prática terapêutica é essencial que o profissional enfermeiro assuma a condição de apropriação de algumas práticas integrativas e complementares e coloque-as em prática não somente em sua vida, mas também em benefício da sociedade (BARBOSA, et. al, 2011).

É de grande relevância salientar que é notória a interação das terapias e a enfermagem. Assim, diante das literaturas exploradas para elaboração deste estudo muitas autores evidenciaram a necessidade incessante de inserir as disciplinas de terapias alternativas e complementares na graduação, o que facilitará futuramente numa atenção integralizada do cuidado diferenciado (LIMA, 2012).

Segundo Azevedo e Peliocin (2011) as terapias alternativas e complementares vem ganhando espaço no que concerne o cuidado, ou seja, um novo cenário do cuidado vem sendo desenvolvido e isso reflete num campo amplo de atuação no mercado de trabalho, principalmente relacionado ao profissional enfermeiro que está em constante contato com o paciente e é o coadjuvante na assistência prestada ao mesmo.

Sabe-se que pode haver uma ausência de qualificação profissional, então este estudo com base nas literaturas evidenciou o quão é possível relacionar diversas formas de cuidar com o cuidado da enfermagem. Mas para que isso venha à tona é necessário que o profissional enfermeiro e outros 
profissionais da área da saúde busquem a qualificação. A partir deste foco de aprimoramento dos conhecimentos poderão inserir essas práticas não convencionais de saúde às suas atividades profissionais (AZEVEDO, PELICIONI, 2011).

O profissional enfermeiro deverá aprimorar seus conhecimentos nessa nova formação, construir o seu emponderamento nessa nova estratégia do cuidado conseguindo relacionar o cuidado convencional com alternativo e sendo capaz de identificar outras necessidades de saúde do paciente e fazendo jus os princípios do SUS (LIMA, 2012).

A atuação do enfermeiro está mais consistente e tem condições de explorar diversas modalidades terapêuticas no desempenho de sua atividade profissional, colocando em prática alternativas de atenção ao cliente, para que o mesmo exerça 0 papel de sua autonomia e cidadania. Estas alternativas complementares em saúde tornam menos sacrificantes e mais prazerosas o tratamento oferecido (LIMA, 2012).

A resolução 197/97 (COFEN 2010), estabelece e reconhece que as terapias integrativas e complementares em saúde podem ser consideradas como um tipo de qualificação para profissional de enfermagem se especializar, desde que tenham concluído em instituição reconhecida de ensino e com carga horária mínima 360 horas (BRASIL, 2010).

A aplicabilidade das práticas terapêuticas complementares em saúde deve ser inserida na graduação, com incentivo dos docentes a buscarem um ensino mais amplo redefinindo conceitos teóricos e evidências científicas destas abordagens terapêuticas (JUNIOR, et. al, 2016).

O gerenciamento das PICS faz se necessário à participação do enfermeiro na divulgação das possibilidades terapêuticas e preventivas aos usuários dentro da atenção primária, assim tornando o campo mais amplo da assistência de enfermagem dentro da ESF podendo ofertar mão de obra especializada (PENNAFORT, et. al, 2012).

O enfermeiro ao tentar desenvolver qualquer prática diferenciada no seu âmbito de trabalho, a sua forma de pensar e agir fica muitas vezes a mercê da ignorância, sem voz com quase nenhuma flexibilidade. Seus questionamentos de como agir em um determinado cuidado fica a mercê do outro. Assim na tentativa de modificar e mostrar que o profissional enfermeiro é o coadjuvante e o protagonista da assistência, que o propósito da inserção das práticas alternativa e complementares será de proporcionar ao profissional enfermeiro exercer suas ações com autonomia, respeitando o seu limite de formação e construir novas teorias na maneira do cuidar (BARBOSA, 2011).

\section{CONSIDERAÇÕES FINAIS}

Incluir a PIC de maneira humanizada e integral na ESF é favorecer ao outro a oportunidade de construir a sua própria autonomia em prol da sua saúde. Uma vez inseridas essas práticas na assistência do enfermeiro ele conseguirá visualizar o paciente como um todo, cuidar e assistir integralmente o ser humano de forma holística.

Desta forma o enfermeiro passará avaliar não somente a doença, mas o indivíduo doente e facilitará na detecção do diagnóstico de enfermagem como no planejamento das intervenções que serão aplicados aos pacientes assistido.

Pensando no bem estar do paciente, que este estudo apresenta como proposta de inovar os cuidados de enfermagem analisando a possibilidade do empoderamento da enfermagem na PIC como uma forma de prestar cuidados diferenciados.

Neste sentido, este estudo é relevante por contribuir na sensibilização do enfermeiro que é essencial no exercício de sua profissão a busca de novas especializações e capacitações, pois a aprendizagem é constante e os desafios sempre virão e é necessários enfrentá-los com criticidade e autonomia. 
Estudos evidenciaram a necessidade de incrementar as práticas interativas complementares às práticas de enfermagem, pois a realidade do atendimento em saúde, atualmente, ainda necessita de um cuidado individualizado, humanizado e qualificado ao usuário que necessita de cuidado e apoio.

\section{REFERÊNCIAS}

1. AZEVEDO E, PELICIONI MCF. Práticas integrativas e complementares de desafios para a educação. Trabalho, Educação e Saúde, 2011; 9(3): 361-378.

2. BARBOSA AM, FERREIRA JDC, LIMA MRG et al. Práticas Alternativas e complementares: ampliando o cuidado em atenção básica, 2011.

3. BRASIL. Ministério da Saúde. Portaria № 145, de 11 de janeiro de 2017, Brasília, 2017.

4. BRASIL. Ministério da Saúde. Portaria № 971, de 03 de maio de 2006. Brasília, 2006.

5. BRASIL. Ministério da Saúde. Cadernos de Atenção Básica. Práticas integrativas e complementares. Plantas medicinais e fitoterapia na atenção básica. Brasília, 2012.

6. CAMPOS RTO, FERRER AL, GAMA CAP et al. Avaliação da qualidade do acesso na atenção primária de uma grande cidade brasileira na perspectiva dos usuários. Saúde Debate, 2014; 38(especial): 252-264.

7. COFEN. Resolução 197/97 de março de 1997. Brasília, DF; 1997.

8. FONTANELLA F, SPECK FP, PIOVEZZAN AP et.al. Conhecimento, acesso e aceitação das praticas integrativas e complementares em saúde por uma comunidade usuária do Sistema Único de Saúde na cidade de Tubarão/SC. Arquivos Catarinenses de Medicina, 2007; 36(2): 69-74.

9. SOUSA IMC, BODSTEIN RCA, TESSER CD. Práticas integrativas e complementares: oferta e produção de atendimentos no SUS e em municípios selecionados. Cadernos de Saúde Pública, 2012; 28(11): 2143- 2154.

10. JUNIOR ET. Práticas Integrativas e Complementares em saúde, uma nova eficácia. Estudos Avançados, 2016; 30(86): 99-112.

11. LIMA KMSV, SILVA KL, TESSER CD. Práticas Integrativas e complementares e a promoção da Saúde: avanços e desafios de um serviço municipal de saúde. Interface: Comunicação, Saúde e Educação, 2012.

12. MELO SCC, SANTANA RG, SANTOS DC et al. Práticas complementares de saúde e os desafios de sua aplicabilidade no hospital: visão de enfermeiros. Revista Brasileira de Enfermagem, 2013; 66(6): 840-846.

13. JÚNIOR ET. Práticas Integrativas e Complementares em Saúde - Blucher Medical Proceedings, 2014; 1 (2): 10.

14. PENNAFORT VPS, FREITAS CHA, JORGE MSB et al. Práticas integrativas e o empoderamento da enfermagem. Revista Mineira de Enfermagem, 2012; 16(2): 289-295.

15. THIAGO SCS, TESSER CD. Percepção de médicos e enfermeiros da Estratégia de Saúde da Família sobre terapias complementares. Revista de Saúde Pública, 2010; 45(2): 249-257. 